ARTIGO ORIGINAL ORIGINAL ARTICLE

\section{Impacto orçamentário de natalizumabe para o tratamento em primeira linha da esclerose múltipla remitente-recorrente altamente ativa sob a perspectiva do sistema de saúde privado brasileiro}

\author{
Budget impact analysis of the adoption of natalizumab \\ as first-line treatment of highly active relapsing-remitting \\ multiple sclerosis from a Brazilian private payer perspective
}

Jéssica Rigolon', Antônio Artur Alves Silva', Ricardo Papaléo Rosim¹

DOI: $10.21115 / J B E S . v 11 . n 2 . p 128-34$

\section{Palavras-chave:}

natalizumabe, esclerose múltipla, impacto orçamentário, sistema de saúde suplementar

\section{Keywords:}

natalizumab, multiple sclerosis, budget impact, private healthcare system

\section{RESUMO}

Objetivos: Os objetivos deste estudo são calcular o custo de um surto de esclerose múltipla sob a perspectiva de uma operadora de saúde privada e o impacto orçamentário da adoção de natalizumabe em primeira linha para esclerose múltipla remitente-recorrente altamente ativa (EMRRAA). Métodos: Para o cálculo do custo do surto, duas abordagens foram adotadas: para surtos que não levam a hospitalizações, foi aplicada uma pesquisa a 33 médicos neurologistas para identificação do consumo de recursos. Microcusteio foi realizado com base em bases de dados públicas. Para o cálculo do custo de surtos que levam a hospitalizações, foi utilizada uma base de contas médicas. Para o cálculo do impacto orçamentário, foi construído um modelo baseado em prevalência. Foram assumidos os seguintes custos: custo de aquisição de natalizumabe, custo de infusão de natalizumabe e custo de surtos. Taxa de ocorrência de surtos para natalizumabe e para seus comparadores, disponível apenas no sistema público, foi obtida em estudos clínicos e metanálises. O caso-base foi realizado considerando-se uma operadora que atende 100 mil vidas na região Sudeste. Análise de sensibilidade foi realizada. Resultados: O custo calculado de um surto foi de R\$ 14.157,21. O impacto orçamentário calculado para adoção de natalizumabe para EMRRAA foi de R\$ 0,64 por beneficiário por ano, ou 0,02\% das despesas assistenciais de uma operadora de saúde suplementar, ou $0,02 \%$ de suas receitas de contraprestações. A análise de sensibilidade confirma que o impacto não chega a 1 real por beneficiário por ano e atinge, no máximo, 0,03\% das despesas assistenciais. Conclusão: Dados os altos benefícios clínicos de natalizumabe, o impacto orçamentário de sua adoção para primeira linha de EMRRAA é considerado baixo. O impacto pode estar superestimado, visto que não foram considerados custos de progressão da doença.

\begin{abstract}
Objetivos: The goals of this study are to evaluate the cost of a multiple sclerosis relapse and the budget impact of adopting natalizumab as first-line therapy for HARRMS, both from a private payer perspective. Methods: For calculating the cost of a relapse, two approaches were adopted: for relapses not resulting in hospitalizations, a research with 33 physicians was made to obtain resource utilization data. Microcosting was performed using public data sources. For calculating costs of relapses leading to hospitalizations, we analyzed a claims database. To calculate the budget impact of adopting natalizumab as per its label indication, we built a prevalence-based model. The following costs were included: drug acquisition, drug infusion and relapses costs. The relapses rates for natalizumab and its comparators present in the public system were calculated based on clinical trials and meta-analysis. The base case was calculated assuming a hypothetical payer covering one hundred lives in the southeastern region of Brazil. Sensitivity analysis was performed. Results: The calculated
\end{abstract}

Recebido em: 28/06/2019. Aprovado para publicação em: 21/07/2019.
1. Biogen, São Paulo, SP, Brasil.
Instituição onde o trabalho foi realizado: Biogen Brasil.
Financiamento: Este estudo foi financiado pela Biogen Brasil.
Autor correspondente: Ricardo Papaléo Rosim. Rua Funchal, 418, 70 andar, Vila Olímpia, São Paulo, SP, Brasil. CEP: 04551-065.
Telefone: +55 (11) 94519-8934. E-mail: ricardo.rosim@biogen.com 
relapse cost was $\mathrm{R} \$ 14,157.21$. The calculated budget impact for adopting natalizumab for HARRMS was R\$ 0.64 per person per year, or $0.02 \%$ of the payer's healthcare expenditures, or $0.02 \%$ of its revenue. The sensibility analysis confirmed that the budget impact does not reach one real per person per year and does not exceed $0.03 \%$ of healthcare expenses. Conclusion: Given the high clinical benefits of adopting Tysabri, its budget impact can be considered low. The results might be overestimated, since disability progression costs were not accounted in the calculations.

\section{Introdução}

A esclerose múltipla (EM) é uma doença autoimune, crônica, progressiva e incapacitante do sistema nervoso central (SNC). Tem etiologia multifatorial, com influência de fatores tanto ambientais como genéticos (Baecher-Allan et al., 2018; Kamm et al., 2014). A doença é caracterizada pela inflamação do SNC, acompanhada de destruição da bainha de mielina e degeneração axonal (Baecher-Allan et al., 2018; Kamm et al., 2014), sendo mais comum em mulheres, majoritariamente jovens, entre 20 e 40 anos (Baecher-Allan et al., 2018; Kamm et al., 2014).

No mundo, a EM é a principal causa de incapacidade não traumática em jovens, com prevalência média de 33 casos para cada 100 mil, sendo mais prevalente em países e regiões mais afastados dos trópicos (Belbasis et al., 2015; Kamm et al., 2014; Markowitz, 2013; Vidal-Jordana \& Montalban, 2017). No Brasil, é menos frequente, com prevalência média de 15 para cada 100 mil habitantes, com um número relativo de casos maior nas regiões Sul e Sudeste do país (da Gama Pereira et al., 2015).

Os pacientes com EM podem apresentar diversos sinais e sintomas, que variam de acordo com a localização das lesões no SNC (Compston \& Coles, 2002; Sá, 2012). Entre os principais sintomas, estão fraqueza muscular, fadiga, dificuldade e perda de deambulação, disfunções urinárias, neurite óptica, diplopia e distúrbios cognitivos (Markowitz, 2013).

A EM pode se apresentar em quatro principais fenótipos: (1) síndrome clinicamente isolada ( $\mathrm{SCl}$ ou CIS, do inglês clinically isolated syndrome); (2) EM remitente-recorrente (EMRR); (3) EM secundariamente progressiva (EMSP) ou (4) EM primariamente progressiva (EMPP), de acordo com o início e a evolução do quadro clínico (Baecher-Allan et al., 2018; Vidal-Jordana \& Montalban, 2017).

A EMRR é a forma comum da doença, sendo responsável por $80 \%$ a $85 \%$ dos casos (Steinman, 2014; Vidal-Jordana \& Montalban, 2017). É caracterizada por episódios de exacerbação, denominados surtos, provocados pela desmielinização e inflamação do SNC, seguidos de períodos de remissão, causados pela capacidade de remielinização do cérebro e de compensação da perda axonal (Baecher-Allan et al., 2018; Kalincik, 2015). A cada surto, a capacidade de regeneração do SNC é reduzida, o que faz com que as incapacidades adquiridas com a exacerbação acumulem-se com o tempo e com o curso natural da doença (Markowitz, 2013). Para avaliar a progressão da incapacidade causada pela EM, a metodologia mais utilizada é a Escala Expandida de Estado de Incapacidade (EDSS, do inglês Expanded Disability Status Scale), que consiste em pontuar a avaliação neurológica em uma escala de 20 pontos, de 0 (exame neurológico normal) a 10 (morte em decorrência de EM) (Amato \& Portaccio, 2007; Kurtzke, 1983).

A importância de se iniciar precocemente o tratamento da EM é amplamente reconhecida, uma vez que o tratamento precoce reduz expressivamente o risco de progressão da doença e retarda o acúmulo de incapacidades (Bergamaschi et al., 2012; Cocco et al., 2015; Kavaliunas et al., 2017). Atualmente, diversas drogas modificadoras da doença (DMDs) estão disponíveis para o tratamento da EM, em sua maioria para tratar EMRR, visando à redução da inflamação no SNC (Garg \& Smith, 2015; Smith et al., 2017). Por tratar-se de uma doença heterogênea, com atividades, velocidades de progressão e localizações de lesões diferentes, o tratamento da EM não é único e deve ser individualizado (Montalban et al., 2018).

Quando se fala em atividade da doença, em seu curso natural, a EM pode ser ativa ou altamente ativa (EMRRAA). As principais características da EMRRAA são surtos frequentes e graves, recuperação incompleta da incapacidade gerada pelos surtos, prejuízo precoce das funções físicas e cognitivas e alta atividade radiológica, com aumento das lesões T2 e gadolínio-positivas (Gd+) (Díaz et al., 2019). Estima-se que 22\% dos pacientes com EMRR tenham a forma altamente ativa da doença (Hutchinson et al., 2009).

Considerando os diferentes perfis de atividade da doença, protocolos recentes desenvolvidos por sociedades médicas nacionais e internacionais passaram a considerar o grau de atividade da doença para a definição do tratamento adequado do paciente (Comini-Frota et al., 2017; Limmroth, 2014; Sorensen, 2014).

No Brasil, oito terapias são reembolsadas no âmbito federal, pelo Sistema Único de Saúde (SUS), sendo três betainterferonas, acetato de glatirâmer e teriflunomida como primeira e segunda linha de tratamento, fumarato de dimetila como segunda linha, fingolimode tanto em segunda como em terceira linha e natalizumabe como opção de tratamento após fingolimode ou em caso de contraindicação ao uso do último (Brasil, 2018). No sistema de saúde suplementar, apenas o natalizumabe tem reembolso obrigatório em âmbito nacional, após falha terapêutica com outras DMDs.

Apesar do grande número de terapias disponíveis, nem o protocolo federal nem a cobertura obrigatória do sistema privado contemplam terapia específica para pacientes 
virgens de tratamento com a doença altamente ativa, que requerem um tratamento precoce com terapias de alta eficácia, como natalizumabe (Díaz et al., 2019). Esse medicamento demonstrou ser altamente efetivo na redução de frequência de surtos e de progressão da doença, por meio de estudos clínicos, metanálises e estudos de mundo real (Fogarty et al., 2016; Hutchinson et al., 2009).

Estudos de impacto econômico da doença vêm sendo desenvolvidos no mundo. Essas análises mostram aumento significativo no custo da doença com a progressão dos escores de EDSS (Flachenecker et al., 2017; Karampampa et al., 2012; Kobelt et al., 2017; Sá et al., 2017). Além disso, os custos de tratamento de pacientes com surtos são maiores em relação a pacientes com a doença controlada (da Silva et al., 2016; Kobelt et al., 2018). No Brasil, existem dois estudos de custo da doença, porém ambos sob a perspectiva do SUS. Até onde sabemos não há nenhum estudo para o sistema privado de saúde, tampouco com foco em doença com alta atividade.

Este estudo tem como objetivo calcular o custo médio de um surto de EM, além de demonstrar, sob a perspectiva do sistema privado de saúde, o impacto orçamentário da ampliação da cobertura de natalizumabe para pacientes virgens de tratamento com a doença altamente ativa.

\section{Métodos}

\section{Número de pacientes e dinâmica populacional}

A análise foi realizada sob a perspectiva de operadoras de saúde privadas no Brasil e o caso-base foi realizado utilizando como parâmetros uma operadora hipotética com 100 mil vidas cobertas operando na região Sudeste do Brasil.

Os pacientes com EMRR dentro dessa operadora hipotética foram estratificados em quatro grupos: pacientes em primeira linha com e sem alta atividade da doença e pacientes em segunda linha ou posterior com e sem alta atividade da doença.

Foram empregados tanto dados epidemiológicos obtidos por meio de busca em literatura quanto dados de mundo real para o cálculo do número de pacientes por segmento, cálculo de crescimento populacional e estimativa de taxa de transição entre os segmentos.

Para o cálculo de pacientes prevalentes, utilizou-se taxa de prevalência de 17 a 18 casos a cada 100 mil habitantes, observada na região Sudeste (Tabela 1). Ao resultado obtido, foi aplicado um fator de $80 \%$, referente à proporção de casos de EMRR entre o total dos casos de EM (Compston \& Coles, 2002). A proporção de pacientes com alta atividade de doença é estimada em 22\% (Hutchinson et al., 2009). Estima-se, com base em dados do Departamento de Tecnologia do SUS (Datasus) e em dados de vendas de medicamentos, que atualmente existam cerca de 17.900 pacientes com EM sendo tratados no SUS, o que representa $57 \%$ da prevalência estimada da doença. Portanto, assume-se que $57 \%$ do número estimado de pacientes sejam tratados.
Tabela 1. Prevalência de esclerose múltipla por 100 mil habitantes por região do Brasil

\begin{tabular}{lccc}
\hline Região & $\begin{array}{c}\text { Valor mínimo } \\
\text { relatado }\end{array}$ & $\begin{array}{c}\text { Valor máximo } \\
\text { relatado }\end{array}$ & $\begin{array}{c}\text { Valor } \\
\text { utilizado }\end{array}$ \\
\hline Centro-Oeste & 10 & 13 & 11,5 \\
\hline Nordeste & 8 & 10 & 9,0 \\
\hline Norte & 4 & 5 & 4,5 \\
\hline Sudeste & 17 & 18 & 17,5 \\
\hline Sul & 26 & 26 & 26,0 \\
\hline
\end{tabular}

Fonte: Comitê Brasileiro de Tratamento e Pesquisa em Esclerose Múltipla.

Para o crescimento anual da população na saúde suplementar, assumiu-se uma taxa de crescimento similar ao crescimento da população brasileira, estimada em 0,9\% e baseada em projeções do Instituto Brasileiro de Geografia e Estatística (IBGE).

A proporção de pacientes em segunda linha ou posteriores de tratamento foi estimada com base em pesquisa de mercado com médicos especialistas, que demonstrou que $44 \%$ dos pacientes já fizeram troca de medicamento.

Não foram assumidas taxas de abandono de tratamento, dada a inexistência de outros medicamentos de cobertura obrigatória na saúde suplementar. Sabe-se que os pacientes inevitavelmente abandonarão o tratamento, seja por escolha do médico, eventos adversos, perda de segurança ou morte, portanto o resultado desta análise pode superestimar o impacto orçamentário.

O horizonte de tempo da análise é de cinco anos.

\section{Custos assumidos}

Foram assumidos os custos de aquisição de natalizumabe, único medicamento de cobertura obrigatória na saúde suplementar para o tratamento da EMRR e objeto desta análise, e custos decorrentes da ocorrência de surtos. Assume-se que, mesmo para pacientes com plano de saúde privado, outros medicamentos seriam dispensados no SUS e os surtos seriam tratados na saúde suplementar.

Para o cálculo do custo de natalizumabe, assume-se o seu preço de fábrica com 18\% de Imposto sobre Circulação de Mercadorias e Serviços (ICMS), alíquota praticada em estados que concentram a maior parte da população brasileira: R\$ 5.413,88 (Câmara de Regulação do Mercado de Medicamentos - CMED). O regime posológico, previsto em bula, é de uma dose a cada quatro semanas, que resulta em 13 doses anuais. O custo anual de tratamento assumido é de $\mathrm{R} \$ 70.380,44$.

Para o cálculo dos custos relativos à ocorrência de surtos que não levam à hospitalização, foi aplicado um questionário com 33 especialistas com o objetivo de identificar a proporção e a quantidade média de recursos médicos utilizados no tratamento de surtos de EM. O resultado desta pesquisa foi utilizado para calcular os custos de surtos que não requerem hospitalizações. Para determinar o cálculo dos custos rela- 
tivos ao tratamento hospitalar dos pacientes em surto, foi utilizada uma base de dados de contas médicas fornecidas pela empresa Orizon, que administra contas de operadoras de saúde suplementar brasileiras, capaz de capturar dados hospitalares mais dificilmente coletáveis por meio de questionário. Para a identificação de surtos de EM que levam a hospitalizações, foram selecionados eventos hospitalares em que houve relato do CID G35 e utilização de corticosteroide endovenoso. Considerou-se que todos os eventos com relato do CID G35 ocorridos em até 30 dias após a hospitalização estariam relacionados ao mesmo surto. Foram excluídos dos cálculos de custo de surtos os custos referentes a medicações modificadoras do curso da doença.

A proporção de surtos que levam à hospitalização também foi coletada na pesquisa com especialistas.

Os custos de infusão de natalizumabe foram obtidos por meio de análise da base de dados da Orizon, sendo estabelecidos em $\mathrm{R} \$ 364$.

A taxa anualizada assumida de ocorrência de surtos para a população em geral com EMRR foi de 0,51 (Einarson et al., 2017). Já para a população com alta atividade da doença, foram assumidas duas diferentes taxas anualizadas: uma para a população em uso de natalizumabe, derivada do estudo AFFIRM (Hutchinson et al., 2009), igual a 0,28, e outra para a população em uso de qualquer medicamento disponível em primeira linha no SUS, conforme Protocolos Clínicos e Diretrizes Terapêuticas (PCDT). Para esta, aplicou-se um risco relativo de 0,7, média dos riscos relativos de taxa anualizada de surtos de terapias de plataforma e teriflunomida observados na metanálise de Fogarty et al. (Fogarty et al., 2016), à taxa do braço placebo do estudo AFFIRM (Hutchinson et al., 2009), resultando em uma taxa anualizada de surtos de 1,02.

Custos relacionados à progressão da incapacidade não foram contemplados nesta análise.

\section{Métricas de avaliação do impacto orçamentário}

O impacto orçamentário da inclusão de natalizumabe para a primeira linha em pacientes com doença altamente ativa foi avaliado de quatro formas diferentes:

- Valor absoluto do impacto orçamentário;

- Impacto por beneficiário por mês;

- Relevância percentual do impacto nas despesas assistenciais per capita da operadora;

- Relevância percentual do impacto nas receitas de contraprestação per capita da operadora.

As despesas assistenciais e receitas de contraprestação per capita referentes ao ano de 2018, último ano completo com dados relatados pela Agência Nacional de Saúde Suplementar (ANS), são apresentadas na Tabela 2.

\section{Análise de sensibilidade}

Além do caso-base, para uma operadora de 100 mil vidas na região Sudeste, serão simulados os resultados para operadoras nas cinco regiões do país, com os seguintes portes: até 100 mil beneficiários, de 100 a 300 mil beneficiários, de 300 a 600 mil beneficiários e acima de 600 mil beneficiários.

\section{Resultados}

\section{Custo de surto}

O custo médio total calculado de um surto foi de R\$ 14.157,21 (Figura 1). Esse valor foi calculado pela média dos custos dos eventos que levam à hospitalização (54\%) e daqueles que não levam à hospitalização (46\%). Os custos de surtos tratados em ambiente hospitalar têm os seguintes componentes: medicamentos ( $\mathrm{R} \$ 6.745,31 \%$ do valor total), procedimentos (R\$ 5.292, 24\% do valor total), materiais (R\$ 4.327, 20\% do valor total), diárias (R\$3.309, 15\% do valor total) e outros (R\$ 2.046 ou 9\% do valor total). Já para os surtos tratados em ambiente ambulatorial, os seguintes componentes foram

Tabela 2. Despesas assistenciais e receitas de contraprestação por porte de operadora

\begin{tabular}{lcc}
\hline Número de beneficiários & $\begin{array}{c}\text { Despesas } \\
\text { assistenciais } \\
\text { médias anuais } \\
\text { per capita } \\
\text { (em reais) }\end{array}$ & $\begin{array}{c}\text { Receitas } \\
\text { médias de } \\
\text { contraprestação } \\
\text { anuais per capita } \\
\text { (em reais) }\end{array}$ \\
\hline Cem a duzentos mil & $2.789,92$ & $3.325,87$ \\
\hline Duzentos a trezentos mil & $2.800,89$ & $3.325,98$ \\
\hline Trezentos a quinhentos mil & $3.276,08$ & $3.872,62$ \\
\hline Quinhentos mil a um milhão & $3.783,69$ & $4.312,58$ \\
\hline Superior a um milhão & $3.696,78$ & $4.394,33$ \\
\hline
\end{tabular}

Fonte: Tabnet ANS.

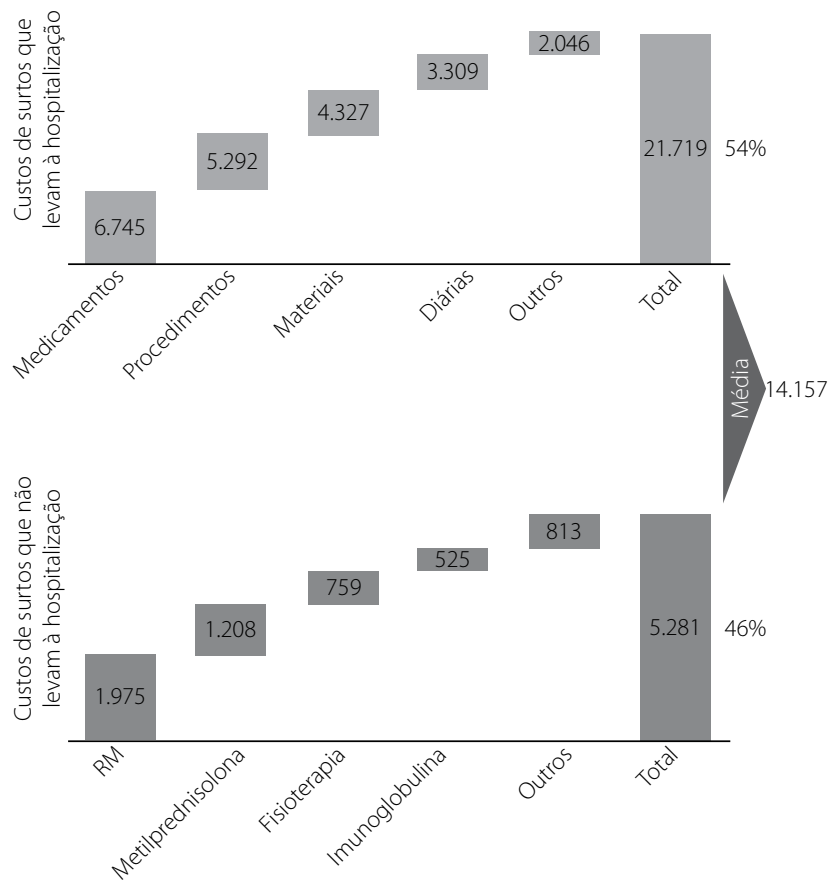

Figura 1. Custo de surto em reais (custos de metilprednisolona e imunoglobulina incluem custos de infusão). 
identificados: ressonância magnética (R\$ 1.975, 37\% do valor total), metilprednisolona endovenosa (R\$ 1.208, 23\% do valor total), fisioterapia (R\$759, 14\% do valor total), imunoglobulina endovenosa ( $R \$ 525,10 \%$ do valor total) e outros ( $R \$ 813,15 \%$ do valor total). Para os itens metilprednisolona e imunoglobulina, o valor considerado contempla também os custos de infusão. Nos casos de surtos que não requerem hospitalizações, foram desconsiderados custos de medicamentos orais, que não são de cobertura obrigatória pelas operadoras de saúde suplementar.

\section{Impacto orçamentário}

Projeta-se que, ao fim dos cinco anos, 8,2 pacientes estejam em tratamento com EMRR, sendo 1,0 em primeira linha com doença altamente ativa (Figura 2).

O custo total, por ano, decorrente da aquisição de natalizumabe varia de $\mathrm{R} \$ 246$ mil a $\mathrm{R} \$ 255$ mil no cenário em que a operadora apenas siga apenas a obrigatoriedade de cobertura de natalizumabe, enquanto no cenário em que se cobre

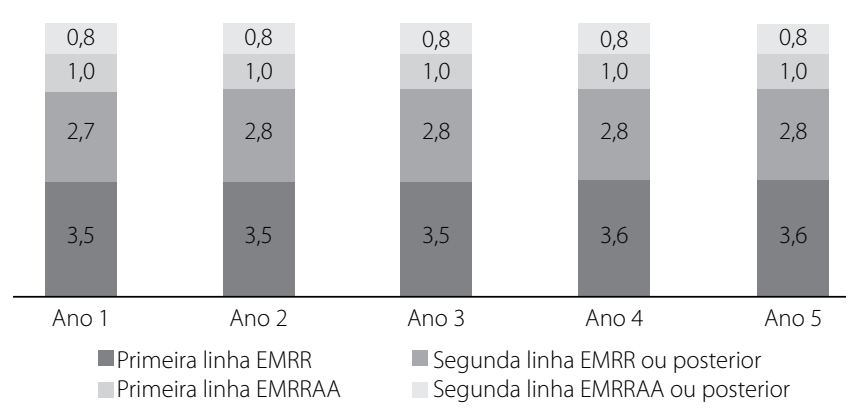

Figura 2. Número projetado de pacientes por ano. também o tratamento para pacientes virgens de tratamento com doença altamente ativa varia de $\mathrm{R} \$ 315$ mil a $\mathrm{R} \$ 326$ mil.

Por outro lado, a cobertura expandida de natalizumabe reduz em $17 \%$ os custos relacionados ao total de surtos dos pacientes da operadora e em $73 \%$ os custos de surtos em pacientes em primeira linha com alta atividade da doença.

Por fim, o impacto orçamentário total em cinco anos é de $\mathrm{R} \$ 321,9$ mil, ou R\$ 0,64 por beneficiário por ano, o que representa $0,023 \%$ das despesas assistenciais da operadora e 0,019\% da mensalidade paga pelos beneficiários.

\section{Análise de sensibilidade}

Ao realizar a análise por grupo de operadora, com base em região e porte, observa-se que o custo incremental da ampliação de uso de natalizumabe não passa de 0,03\% das despesas assistenciais, podendo chegar a 0,01\% em operadoras de grande porte na região Nordeste do país, conforme a Tabela 4.

O impacto orçamentário, em nenhum grupo de operadoras, chega a $\mathrm{R} \$ 1,00$ por beneficiário por ano.

\section{Discussão e conclusões}

Este estudo demonstra, de forma fidedigna, que o impacto da utilização de natalizumabe como primeira linha no tratamento da EMRR altamente ativa é irrisório quando comparado às despesas já consumidas com cuidados à saúde dos beneficiários de planos de saúde.

Soma-se a isso a alta necessidade dos pacientes com essa agressiva forma da doença em receber de forma precoce uma terapia altamente eficaz (Bergamaschi et al., 2012; Cocco et al., 2015; Kavaliunas et al., 2017), atualmente dispensada apenas para pacientes que já passaram por outros

Tabela 3. Impacto orçamentário da adoção de natalizumabe em primeira linha de tratamento da EMRRAA (em reais)

\begin{tabular}{|c|c|c|c|c|c|c|}
\hline & Ano 1 & Ano 2 & Ano 3 & Ano 4 & Ano 5 & Total \\
\hline $\begin{array}{l}\text { Sem adoção de natalizumabe } \\
\text { para primeira linha de EMRRAA }\end{array}$ & $324.400,72$ & $327.320,32$ & $330.266,21$ & $333.238,60$ & $336.237,75$ & $1.651 .463,60$ \\
\hline Natalizumabe & $245.956,55$ & $248.170,16$ & $250.403,69$ & $252.657,33$ & $254.931,24$ & $1.252 .118,99$ \\
\hline Infusão & $16.536,79$ & $16.685,62$ & $16.835,79$ & $16.987,31$ & $17.140,20$ & $84.185,71$ \\
\hline Surto & $61.907,37$ & $62.464,54$ & $63.026,72$ & $63.593,96$ & $64.166,31$ & $315.158,91$ \\
\hline $\begin{array}{l}\text { Adoção de natalizumabe para } \\
\text { EMRRAA para primeira linha de } \\
\text { EMRRAA }\end{array}$ & $387.647,67$ & $391.136,50$ & $394.656,73$ & $398.208,64$ & $401.792,52$ & $1.973 .442,07$ \\
\hline Natalizumabe & $314.824,39$ & $317.657,81$ & $320.516,73$ & $323.401,38$ & $326.311,99$ & $1.602 .712,30$ \\
\hline Infusão & $21.167,09$ & $21.357,59$ & $21.549,81$ & $21.743,76$ & $21.939,45$ & $107.757,70$ \\
\hline Surto & $51.656,19$ & $52.121,10$ & $52.590,19$ & $53.063,50$ & $53.541,07$ & $262.972,06$ \\
\hline Impacto orçamentário & $63.246,96$ & $63.816,18$ & $64.390,52$ & $64.970,04$ & $65.554,77$ & $321.978,47$ \\
\hline Impacto por beneficiário por ano & 0,63 & 0,64 & 0,64 & 0,65 & 0,66 & 0,64 \\
\hline $\begin{array}{l}\text { Impacto como proporção das } \\
\text { despesas assistenciais }\end{array}$ & $0,02 \%$ & $0,02 \%$ & $0,02 \%$ & $0,02 \%$ & $0,02 \%$ & $0,02 \%$ \\
\hline $\begin{array}{l}\text { Impacto como proporção da } \\
\text { receita de contraprestação }\end{array}$ & $0,02 \%$ & $0,02 \%$ & $0,02 \%$ & $0,02 \%$ & $0,02 \%$ & $0,02 \%$ \\
\hline
\end{tabular}


Tabela 4. Impacto orçamentário por classificação de operadora

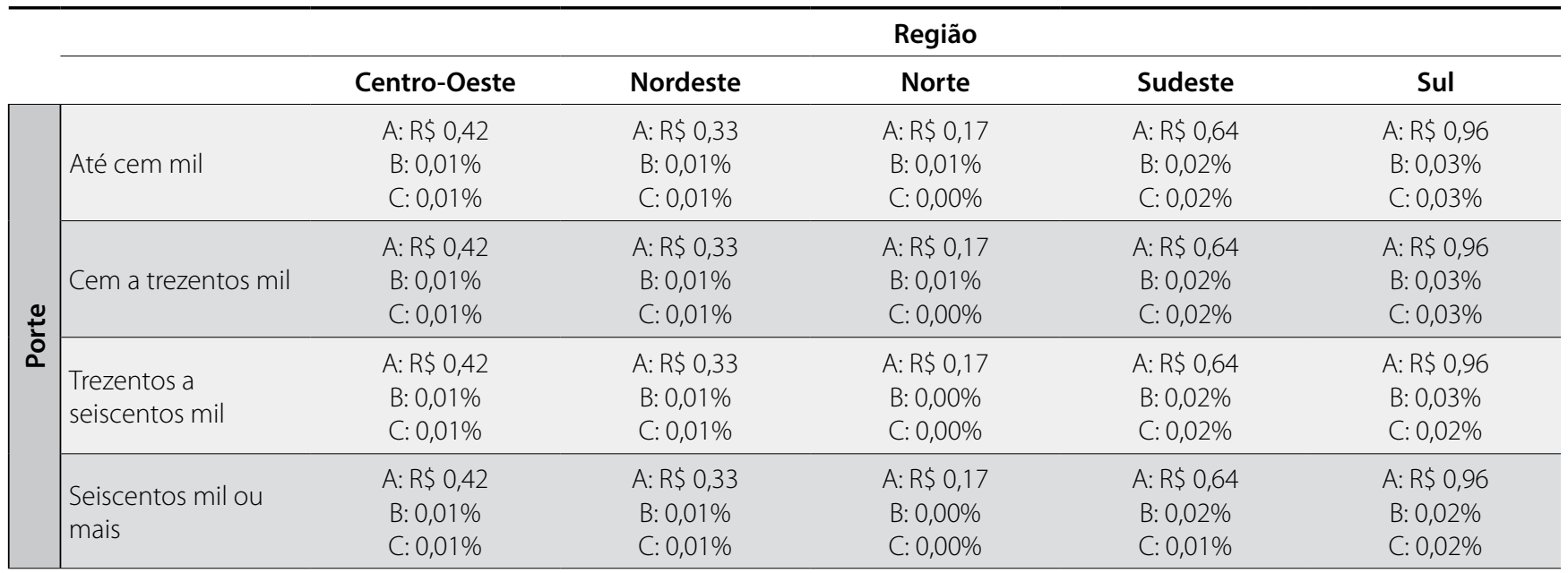

A: impacto por beneficiário por ano; B: impacto em relação às despesas assistenciais; C: impacto em relação à receita de contraprestação.

tratamentos, tanto no sistema privado quanto no sistema público de saúde.

As limitações do estudo são decorrentes especialmente da falta de dados disponíveis na literatura e, em geral, tendem a superestimar o impacto orçamentário calculado, e não alteram suas conclusões. As limitações incluem: falta de taxa de abandono de tratamento e premissa de troca de linha de tratamento idêntica entre pacientes com doença altamente ativa e população em geral.

Adicionalmente, deve-se ressaltar que a análise não leva em conta custos gerados pela progressão da doença e consequente aumento de incapacidade. O natalizumabe, por ser um medicamento mais efetivo para a população com alta atividade da doença do que os medicamentos de primeira linha presentes no SUS (Prosperini et al., 2017), seria capaz de gerar redução de custos não contemplada nessa análise.

Balanceando a alta eficácia do natalizumabe (Hutchinson et al., 2009; Prosperini et al., 2017), a importante necessidade médica não atendida, os potenciais custos evitados de longo prazo (Kobelt et al., 2019) e o baixo impacto orçamentário, conclui-se que a cobertura expandida de natalizumabe além da determinada pela Diretriz de Utilização da ANS, de forma que os pacientes com alta atividade da doença o recebam em primeira linha, pode trazer alto benefício clínico aos pacientes e profissionais de saúde com baixa carga financeira à fonte pagadora.

\section{Agradecimentos}

Agradecemos aos 33 neurologistas que responderam à pesquisa sobre utilização de recursos de saúde em surtos de esclerose múltipla.

Agradecemos, especialmente, à doutora Yara Fragoso pelo envio do questionário aos especialistas e pela consultoria técnica na confecção deste estudo.

\section{Referências bibliográficas}

Amato MP, Portaccio E. Clinical outcome measures in multiple sclerosis. J Neurol Sci. 2007;259(1-2);118-22.

Baecher-Allan C, Kaskow BJ, Weiner HL. Multiple Sclerosis: Mechanisms and Immunotherapy. Neuron. 2018;97(4):742-68.

Belbasis L, Bellou V, Evangelou E, loannidis JPA, Tzoulaki I. Environmental risk factors and multiple sclerosis: an umbrella review of systematic reviews and meta-analyses. Lancet Neurol. 2015;14(3):263-73.

Bergamaschi R, Quaglini S, Tavazzi E, Amato MP, Paolicelli D, Zipoli V, et al. Immunomodulatory therapies delay disease progression in multiple sclerosis. Mult Scler. 2012;22(13):1732-40.

Brasil. Ministério da Saúde. Portaria Conjunta no 10, de 02 de abril de 2018. Aprova o Protocolo Clínico e Diretrizes Terapêuticas da Esclerose Múltipla. Brasília; 2018

Cocco E, Sardu C, Spinicci G, Musu L, Massa R, Frau J, et al. Influence of treatments in multiple sclerosis disability: a cohort study. Mult Scler 2015;21(4):433-41.

Comini-Frota ER, Vasconcelos CCF, Mendes MF. Guideline for multiple sclerosis in Brazil: Consensus from the neuroimmunology Scientific Department of the Brazilian Academy of Neurology. Arq NeuroPsiquiatr. 2017;75(1):57-65.

Compston A, Coles A. Multiple sclerosis. Lancet. 2002;359(9313):1221-31.

da Silva NL, Takemoto MLS, Damasceno A, Fragoso YD, Finkelsztejn A, Becker $\mathrm{J}$, et al. Cost analysis of multiple sclerosis in Brazil: a cross-sectional multicenter study. BMC Health Serv Res. 2016;16:102.

Díaz C, Zarco LA, Rivera DM. Highly active multiple sclerosis: an update. Mult Scler Relat Disord. 2019;30:215-24.

Einarson TR, Bereza BG, Machado M. Comparative effectiveness of interferons in relapsing-remitting multiple sclerosis: a meta-analysis of real-world studies. Curr Med Res Opin. 2017;33(3):579-93.

Flachenecker P, Kobelt G, Berg J, Capsa D, Gannedahl M; European Multiple Sclerosis Platform. New insights into the burden and costs of multiple sclerosis in Europe: Results for Germany. Mult Scler. 2017;23(2 Suppl):78-90.

Fogarty E, Schmitz S, Tubridy N, Walsh C, Barry M. Comparative efficacy of disease-modifying therapies for patients with relapsing remitting multiple sclerosis: Systematic review and network meta-analysis. Mult Scler Relat Disord. 2016;9:23-30. 
Garg N, Smith TW. An update on immunopathogenesis, diagnosis, and treatment of multiple sclerosis. Brain Behav. 2015;5(9):e00362.

Hutchinson M, Kappos L, Calabresi PA, Confavreux C, Giovannoni G, Galetta SL, et al.; AFFIRM and SENTINEL Investigators. The efficacy of natalizumab in patients with relapsing multiple sclerosis: subgroup analyses of AFFIRM and SENTINEL. J Neurol. 2009;256(3):405-15.

Kalincik T. Multiple Sclerosis Relapses: Epidemiology, Outcomes and Management. A Systematic Review. Neuroepidemiology. 2015;44(4):199214.

Kamm CP, Uitdehaag BM, Polman CH. Multiple sclerosis: current knowledge and future outlook. Eur Neurol. 2014;72(3-4):132-41.

Karampampa K, Gustavsson A, Miltenburger C, Eckert B. Treatment experience, burden and unmet needs (TRIBUNE) in MS study: results from five European countries. Mult Scler. 2012;18(2 Suppl):7-15.

Kavaliunas A, Manouchehrinia A, Stawiarz L, Ramanujam R, Agholme J, Hedström AK, et al. Importance of early treatment initiation in the clinical course of multiple sclerosis. Mult Scler. 2017;23(9):1233-40.

Kobelt G, Teich V, Berner S, Canzoneri AM, Cavalcanti M, Gonçalves EM. Burden of Multiple Sclerosis in Brazil. Berlim: ECTRIMS Congress; 2018.

Kobelt G, Teich V, Cavalcanti M, Canzonieri AM. Burden and cost of multiple sclerosis in Brazil. PLoS One. 2019;14(1):e0208837.

Kobelt G, Thompson A, Berg J, Gannedahl M, Eriksson J; MSCOI Study Group; European Multiple Sclerosis Platform. New insights into the burden and costs of multiple sclerosis in Europe. Mult Scler. 2017;23(8):1123-36.

Kurtzke JF. Rating neurologic impairment in multiple sclerosis: an expanded disability status scale (EDSS). Neurology. 1983;33(11):1444-52.

Limmroth V. Treatment of relapsing-remitting multiple sclerosis: current and future algorithms. Eur Neurol. 2014;72 Suppl 1:35-8.
Markowitz CE. Multiple sclerosis update. Am J Manag Care. 2013;19(16 Suppl):S294-300.

Montalban X, Gold R, Thompson AJ, Otero-Romero S, Amato MP, Chandraratna D, et al. ECTRIMS/EAN Guideline on the pharmacological treatment of people with multiple sclerosis. Mult Scler. 2018;24(2):96-120.

da Gama Pereira AB, Sampaio Lacativa MC, da Costa Pereira FF, Papais Alvarenga RM. Prevalence of multiple sclerosis in Brazil: A systematic review. Mult Scler Relat Disord. 2015;4(6):572-9.

Prosperini L, Saccà F, Cordioli C, Cortese A, Buttari F, Pontecorvo S, et al. Real-world effectiveness of natalizumab and fingolimod compared with self-injectable drugs in non-responders and in treatment-naïve patients with multiple sclerosis. J Neurol. 2017;264(2):284-94.

Sá MJ. Physiopathology of symptoms and signs in multiple sclerosis. Arq Neuro-Psiquiatr. 2012;70(9):733-40.

Sá MJ, Kobelt G, Berg J, Capsa D, Dalén J; European Multiple Sclerosis Platform. New insights into the burden and costs of multiple sclerosis in Europe: Results for Portugal. Mult Scler. 2017;23(2 Suppl):143-54.

Smith AL, Cohen JA, Hua LH. Therapeutic Targets for Multiple Sclerosis: Current Treatment Goals and Future Directions. Neurotherapeutics. 2017;14(4):952-60.

Sorensen PS. New management algorithms in multiple sclerosis. Curr Opin Neurol. 2014;27(3):246-59.

Steinman L. Immunology of relapse and remission in multiple sclerosis. Annu Rev Immunol. 2014;32:257-81.

Vidal-Jordana A, Montalban X. Multiple Sclerosis: Epidemiologic, Clinical, and Therapeutic Aspects. Neuroimaging Clin N Am. 2017;27(2):195-204. 